\title{
Development of an Annular Helicon Source for Electric Propulsion Applications
}

\author{
Brian E. Beal ${ }^{*}$ \\ Aerojet-Redmond Operations, Redmond, WA 98052 \\ Alec D. Gallimore ${ }^{\dagger}$, David P. Morris ${ }^{\star}$, and Christopher Davis ${ }^{\S}$ \\ ElectroDynamic Applications, Inc., Ann Arbor, MI 48113 \\ and \\ Kristina M. Lemmer ${ }^{* *}$ \\ University of Michigan, Ann Arbor, MI 48109
}

\begin{abstract}
The performance of typical electrostatic propulsion systems, such as the Hall thruster, is limited in part by inefficiencies in the electron bombardment ionization process. These limitations become especially pronounced at the operating conditions required to achieve high thrust-to-power ratios. One approach for achieving significant increases in efficiency at such operating conditions is to replace the typically-employed DC ionization mechanism with a helicon source, which is widely regarded as an efficient method for creating a highdensity, low-temperature plasma. Standard cylindrical helicons, however, are not amenable to straightforward integration with annular Hall thrusters. A rigorous mathematical treatment of helicon wave physics has been completed to establish the boundary conditions required to create an annular helicon source for both the $m=0$ and $m=1$ azimuthal modes. This analysis reveals no fundamental barriers to creation of an annular helicon source so long as the radial boundary conditions are set appropriately.
\end{abstract}

\section{Nomenclature}

$\vec{B}_{0} \quad=$ DC magnetic flux vector

$B_{0} \quad=$ magnitude of DC magnetic flux

$\vec{B} \quad=$ AC magnetic flux vector

$B_{r}, B_{\theta}, B_{z}=$ radial, azimuthal, and axial components of magnetic field

$c \quad=$ speed of light in vacuum

$\vec{E} \quad=$ electric field vector

$E_{r}, E_{\theta}, E_{z}=$ radial, azimuthal, and axial components of electric field

$e \quad=$ electron charge

$I_{s p} \quad=$ specific impulse

$\vec{j} \quad=$ current density vector

$j_{\perp} \quad=$ current density perpendicular to magnetic field

$J_{m} \quad=$ Bessel function of the first kind, order $\mathrm{m}$

$k=$ axial wave number

$m \quad=$ azimuthal wave mode (integer)

\footnotetext{
${ }^{*}$ Currently with Air Force Research Laboratory, 1 Ara Rd., Edwards AFB, CA 93524, Member AIAA

${ }^{\dagger}$ CEO and Chief Engineer, P.O. Box 131460, Ann Arbor, MI 48113-1460, Associate Fellow AIAA.

* Senior Engineer, P.O. Box 131460, Ann Arbor, MI 48113-1460, Member AIAA

$\S$ Senior Engineer, P.O. Box 131460, Ann Arbor, MI 48113-1460, Member AIAA

** Ph.D Candidate, Aerospace Engineering, 1320 Beal Ave, Ann Arbor, MI 48109, Member AIAA
} 


$\begin{array}{ll}n_{0} & =\text { steady-state electron number density } \\ n & =\text { perturbed electron number density } \\ P_{\text {ionization }} & =\text { ionization power } \\ P_{\text {input }} & =\text { input electrical power } \\ P_{\text {other }} & =\text { power required for ancillary components such as electromagnets, heaters, etc. } \\ P_{\text {thrust }} & =\text { thrust power } \\ r & =\text { radial coordinate } \\ \mathrm{Z} & =\text { axial coordinate } \\ \mathrm{R}_{\mathrm{wall}} & =\text { radial coordinate of a physical boundary } \\ \mathrm{R}_{\text {in }}, \mathrm{R}_{\text {out }} & =\text { radial coordinates of the inner and outer walls of an annular source, respectively } \\ t & =\text { time } \\ \mathrm{T} & =\text { transverse wave number } \\ T / P & =\text { thrust-to-power ratio } \\ \alpha & =\text { total wave number } \\ \eta & =\text { thrust efficiency } \\ \theta & =\text { azimuthal coordinate } \\ \mu_{0} & =\text { permeability of free space } \\ \omega & =\text { angular frequency of wave } \\ \omega_{\mathrm{c}} & =\text { electron cyclotron frequency } \\ \omega_{\mathrm{LH}} & =\text { lower hybrid frequency } \\ \omega_{\mathrm{p}} & =\text { electron plasma frequency } \\ \xi_{\mathrm{q}} & =\text { q }\end{array}$

\section{Introduction}

G lectric propulsion (EP) systems are increasingly being used for orbit topping and stationkeeping maneuvers Conboard modern spacecraft. Due to their high specific impulse compared to chemical propulsion systems, EP devices offer significant reductions in the propellant mass required to perform a given mission. Of the various types of EP, the Hall effect thruster (HET) is often favored for near-Earth applications due to its ability to provide moderately-high specific impulses (typically 1500-2500 seconds) at reasonable electrical efficiencies (generally 50$60 \%$ ). A typical Hall thruster is annular in geometry and consists primarily of an upstream anode, a downstream cathode, and a magnetic circuit. ${ }^{1,2}$ Electrons emitted from the cathode, which is electrically biased to a negative potential, are drawn upstream to the positively biased anode. The motion of these electrons is impeded by a predominantly radial magnetic field established by the magnetic circuit. As electrons migrate toward the anode, a fraction of them collide with propellant atoms, which are injected into the annular space. These collisions result in ionization of the propellant. The ions are then accelerated through the electrostatic field established between the anode and cathode resulting in a high velocity stream of ions.

Due to the desire to make maximum use of the electrical power available for propulsion aboard a given spacecraft, one of the most important figures of merit used to characterize the performance of an EP device is its thrust efficiency. In its most fundamental form, the thrust efficiency can be defined as in Eqn. 1 and, using the expression shown in Eqn. 2, written in the form of Eqn. 3. Considering $P_{\text {other }}$ to be small, Eqn. 3 reveals the intuitively obvious result that the thrust efficiency of an EP device is maximized when the power required for propellant ionization is minimized. In conventional HETs, propellant atoms are ionized via a DC electron bombardment process as described above. Devices relying on such processes are generally reported to result in an ionization cost (i.e. the energy required to create an ion) of approximately ten times the theoretical minimum. ${ }^{3}$ For example, if one assumes that most of the thermal power deposited into a Hall thruster results from inefficiencies in the ionization process, it can be inferred from previously published measurements that the ionization cost in the current state-of-the-art Hall thruster, Aerojet's BPT-4000, is approximately $120 \mathrm{eV}$ for operation on xenon, which has a first ionization potential of $12.1 \mathrm{eV} .{ }^{4}$ This inefficient ionization process limits the efficiency of Hall thrusters at all operating conditions.

$$
\begin{gathered}
\eta \equiv \frac{P_{\text {thrust }}}{P_{\text {input }}} \\
P_{\text {input }} \equiv P_{\text {thrust }}+P_{\text {ionization }}+P_{\text {other }}
\end{gathered}
$$




$$
\eta=\frac{1}{1+\frac{P_{\text {ionization }}+P_{\text {other }}}{P_{\text {thrust }}}}
$$

In addition to limiting the Hall thruster's ultimate efficiency at all operating conditions, the reliance on DC electron bombardment also limits the range of specific impulses over which reasonably efficient operation can be achieved. At high specific impulses, many Hall thrusters show a decrease in efficiency due, in part, to the creation of multiply-charged ions as a result of the increasing electron temperature that occurs at high discharge voltages. Fortunately, this limitation has been largely mitigated by advanced magnetic field topologies that prevent significant increases in ionization cost at high specific impulse operating conditions. ${ }^{5}$ The limitation encountered at low- $\mathrm{I}_{\mathrm{sp}}$ (i.e., high $T / P$, low discharge voltage) operating conditions, on the other hand, is more fundamental and is unlikely to be fully mitigated by straightforward design advances. First, to achieve high $T / P$ a Hall thruster must operate at low discharge voltages and high currents. Operating at high currents requires creation of more ions such that any inefficiencies in the ionization process become a larger fraction of the total power input. Second, electron bombardment ionization occurs only when a neutral propellant atom is struck by an electron traveling with a kinetic energy in excess of the first ionization potential of the propellant atom. For a thermal electron population, the electron velocity distribution is qualitatively similar to the function depicted in Fig. 1. In this figure, the crosshatched areas represent electrons having sufficient energy to cause ionization. Since the ionization potential is a function only of the propellant gas being used, it does not change as a function of thruster operating conditions. Examination of Fig. 1 shows that the fraction of the electron population having energy in excess of the ionization threshold is a function of the width of the electron velocity (or energy) distribution; i.e. the electron temperature. While the factors determining the maximum electron temperature in a typical Hall thruster are complicated, this value can be approximated to be roughly $10 \%$ of the applied discharge voltage for most modern thrusters (Ref. 6 and references therein). It follows that at the low discharge voltages required to provide operation at high $T / P$, only a small fraction of the electron population has sufficient energy to result in propellant ionization. This decreasing fraction of energetic electrons leads to an increase in the effective ionization cost and contributes to the decrease in overall efficiency exhibited by typical Hall thrusters at low specific impulses.

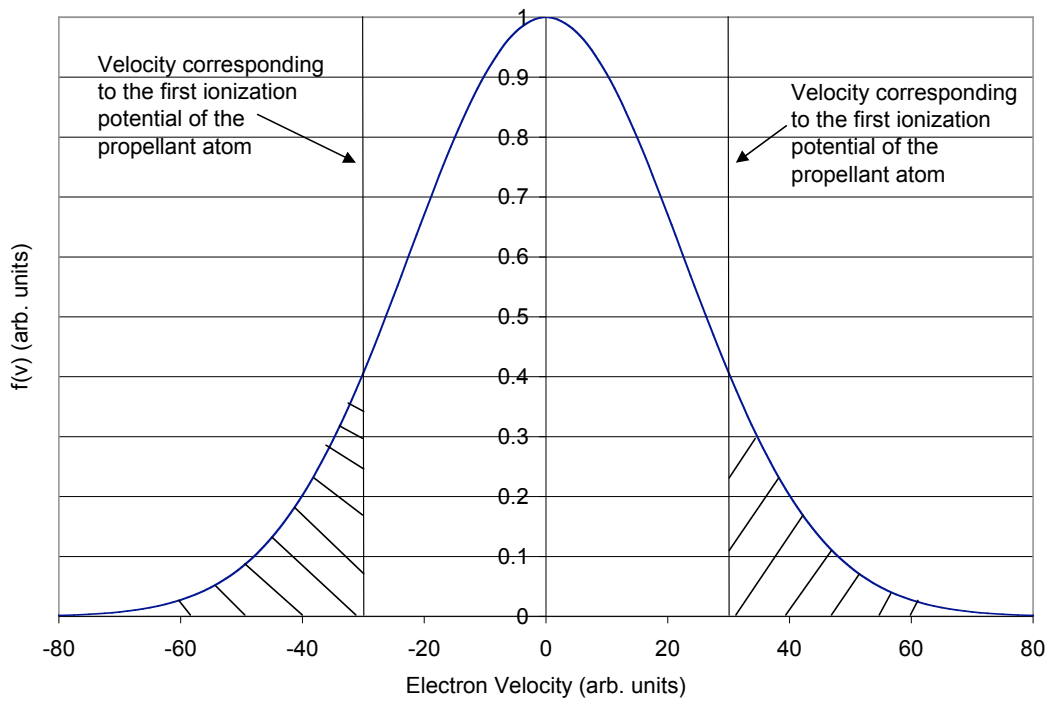

Figure 1. A qualitative depiction of a thermal electron velocity distribution. The cross-hatched areas represent the fraction of the population having sufficient energy to cause ionization.

One approach that may be considered to improve the performance of the Hall thruster, as well as other EP devices, is to replace or supplement the conventional electron bombardment ionization mechanism with a more efficient plasma source. In particular, one device that appears promising is the helicon source, which employs an RF antenna and a static magnetic field to produce cylindrically bounded whistler waves and is widely regarded as the 
most efficient method for producing a high-density, low-temperature plasma. ${ }^{3,7,8}$ For example, helicon sources have been reported to produce approximately an order of magnitude more plasma than a DC electron bombardment source for a given input power. ${ }^{3}$ Helicon sources are thus capable of realizing actual ionization costs approaching the theoretical minimum, which is almost a factor of 10 lower than that obtained in traditional Hall thrusters. Although employing helicon ionization sources is likely to be beneficial for a variety of EP devices, their traditionally cylindrical geometry may prove to be a liability in attempting to integrate them into certain engines such as the annular Hall thruster. In an attempt to expand the geometric configurations in which the benefits of helicon sources may be realized, the analysis below generalizes the derivation of the helicon wave relations and presents the conditions that must be met in order to establish an annular helicon source.

\section{Analysis}

The requirements for propagation of helicon waves in an annular geometry can be accomplished via a "first principles" approach that follows directly from linearized versions of Maxwell's equations. In the derivation below, we follow the classical approach of Chen and deviate from well-established results only to the extent necessary to expand the applicability of the governing equations to annular, rather than cylindrical, boundary conditions. ${ }^{9}$

The properties of helicon waves may be derived starting with the relations shown in Eqns. 4-6 where variables with the subscript 0 represent static quantities while unscripted variables denote perturbed, or wave, quantities. Manipulation of Eqns. 4-6 leads to Eqns. 7-9, where the subscript $\perp$ represents the direction perpendicular to the static magnetic field, which is assumed to be in the axial, $z$, direction by convention. In the derivation of Eqns. 7-9, it has been assumed that the frequency range of interest is high enough that ion motions can be neglected and low enough that electron cyclotron motion can be neglected relative to guiding center motion, as described by Eqn. 10 .

$$
\begin{gathered}
\nabla \times \vec{E}=-\frac{\partial \vec{B}}{\partial t} \\
\nabla \times \vec{B}=\mu_{0} \vec{j} \\
\vec{E}=\frac{\vec{j} \times \vec{B}_{0}}{e n_{0}} \\
\nabla \cdot \vec{B}=0 \\
\nabla \cdot \vec{j}=0 \\
\vec{j}_{\perp}=-\frac{e n_{0} \vec{E} \times \vec{B}_{0}}{B_{0}^{2}} \\
\omega_{L H}<\omega<<\omega_{c}
\end{gathered}
$$

Given the fundamental relations of Eqns. 4-9, the derivation of helicon wave parameters can proceed by assuming perturbations of the form exp [i(m $\theta+\mathrm{kz}-\omega \mathrm{t})]$. Assuming waves of this form and combining Eqns. 4-6 leads to Eqn. 11. Defining the parameter $\alpha$ according to Eqn. 12 and taking the curl of Eqn. 11 results in Eqn. 13, which is the main equation from which subsequent helicon wave relations are derived. Further, by comparing Eqn. 11 with Eqn. 5, one can deduce Eqn. 14, which reveals that the wave current is parallel to the perturbed magnetic field for this type of wave. This point will become important later when boundary conditions are applied to the general relations. 


$$
\begin{gathered}
\vec{B}=\left(\frac{k B_{0}}{\omega \mu_{0} e n_{0}}\right) \nabla \times \vec{B} \\
\alpha \equiv \frac{\omega}{k} \frac{\mu_{0} e n_{0}}{B_{0}}=\frac{\omega}{k} \frac{\omega_{p}^{2}}{\omega_{c} c^{2}} \\
\nabla^{2} \vec{B}+\alpha^{2} \vec{B}=0 \\
\vec{j}=\left(\frac{\alpha}{\mu_{0}}\right) \vec{B}
\end{gathered}
$$

Separating Eqn. 13 into components and formulating the problem in cylindrical coordinates leads to Eqn. 15 for the $\mathrm{z}$ component. Here we have defined $\mathrm{T}$ as shown in Eqn. 16. We note by examination that Eqn. 15 is a form of Bessel's equation, the general solution of which is given by Eqn. 17 where $J_{m}$ and $Y_{m}$ are the Bessel functions of the first and second kind (order $\mathrm{m}$ ), respectively, and $\mathrm{C}_{1}$ and $\mathrm{C}_{2}$ are constants of integration. Because $\mathrm{Y}_{\mathrm{m}}$ diverges at small values of $\mathrm{Tr}$, physically meaningful solutions are generally taken to be those for which $\mathrm{C}_{2}=0$, such that the axial wave magnetic field is given by Eqn. $18 .{ }^{\dagger \dagger}$

$$
\begin{gathered}
r^{2} \frac{\partial^{2} B_{z}}{\partial r^{2}}+r \frac{\partial B_{z}}{\partial r}+\left(r^{2} T^{2}-m^{2}\right) B_{z}=0 \\
T^{2} \equiv \alpha^{2}-k^{2} \\
B_{z}=C_{1} J_{m}(T r)+C_{2} Y_{m}(T r) \\
B_{z}=C_{1} J_{m}(T r)
\end{gathered}
$$

The $\mathrm{r}$ and $\theta$ components of Eqn. 13 can be written as Eqns. 19 and 20, respectively, which can be solved in terms of $\mathrm{B}_{\mathrm{z}}$ and its radial partial derivative. Substituting Eqn. 18 into this result yields Eqns. 21 and 22, which, along with Eqn. 17, define all three components of the wave magnetic field. The wave electric field follows directly from Eqn. 4 and its components are given here for reference as Eqns. 23-25. ${ }^{9}$

$$
\begin{gathered}
\frac{i m}{r} B_{z}-i k B_{\theta}=\alpha B_{r} \\
i k B_{r}-\frac{\partial B_{r}}{\partial r}=\alpha B_{\theta} \\
B_{r}=\frac{i C_{1}}{T^{2}}\left[\frac{m \alpha}{r} J_{m}(T r)+k \frac{\partial J_{m}(T r)}{\partial r}\right]
\end{gathered}
$$

\footnotetext{
${ }^{\dagger}$ Strictly speaking, if one considers only the annular case, it may be possible to generate solutions with $\mathrm{C}_{2} \neq 0$. In order to show the compatibility of the cylindrical and annular solutions, however, we limit our discussion here to the more restrictive case of $\mathrm{C}_{2}=0$, which is the only physically meaningful solution for a cylindrical helicon source.
} 


$$
\begin{gathered}
B_{\theta}=-\frac{C_{1}}{T^{2}}\left[\frac{m k}{r} J_{m}(T r)+\alpha \frac{\partial J_{m}(T r)}{\partial r}\right] \\
E_{r}=\frac{\omega}{k} B_{\theta}=-\frac{C_{1}}{T^{2}} \frac{\omega}{k}\left[\frac{m k}{r} J_{m}(T r)+\alpha \frac{\partial J_{m}(T r)}{\partial r}\right] \\
E_{\theta}=-\frac{\omega}{k} B_{r}=-\frac{i C_{1}}{T^{2}} \frac{\omega}{k}\left[\frac{m \alpha}{r} J_{m}(T r)+k \frac{\partial J_{m}(T r)}{\partial r}\right] \\
E_{z}=0
\end{gathered}
$$

At this point it is worth reiterating that all of the results shown above are universal and are not a function of geometry. In other words, we have made no assumptions that would limit the applicability of the above results to cylindrical rather than annular sources. We can now proceed with the application of boundary conditions by assuming a pair of cylindrical boundaries at arbitrary radii. For an insulating boundary, the condition $\mathrm{j}_{\mathrm{r}}=0$ must hold, and from Eqn. 14 this requires $\mathrm{B}_{\mathrm{r}}=0$. On the other hand, a conducting boundary condition requires $\mathrm{E}_{\theta}=0$, which also requires $\mathrm{B}_{\mathrm{r}}=0$ according to Eqn. 24. Thus, regardless of the nature of the bounding wall, the condition $\mathrm{B}_{\mathrm{r}}=0$ must hold at the physical boundaries of the plasma. From Eqn. 21, we can then establish the boundary condition shown in Eqn. 26 at $\mathrm{r}=\mathrm{R}_{\text {wall }}$.

$$
m \alpha J_{m}\left(T R_{\text {wall }}\right)+k R_{\text {wall }} \frac{\partial J_{m}\left(T R_{\text {wall }}\right)}{\partial r}=0
$$

One can solve Eqn. 26 by first selecting an azimuthal wave mode ( $\mathrm{m}=0$, 1, etc.), which is physically determined by the geometry of the driving antenna. The most common wave modes for helicon sources are the $\mathrm{m}=0$ and $\mathrm{m}=1$ modes. ${ }^{3}$ Examining first the $\mathrm{m}=0$ mode, we see that a nontrivial solution to Eqn. 26 requires that the derivative of the zeroeth order Bessel function must go to zero at the boundaries. By applying the well-known recurrence relation shown in Eqn. 27, this requirement can be written more conveniently as a requirement on the first order Bessel function as shown in Eqn. 28. Eqn. 28 then gives an exact boundary condition for the $m=0$ mode. In general, Eqn. 28 is satisfied for cylindrical helicon sources since $\mathrm{J}_{1}$ goes to zero at $\mathrm{r}=0$ and the bounding cylinder then forces the Bessel function to zero by satisfying the condition $\mathrm{TR}_{\text {wall }}=3.83$ where 3.83 is the first root of $\mathrm{J}_{1}$. The boundary condition thus simply specifies a relation between the transverse wave number, $\mathrm{T}$, and the geometry of the bounding cylinder. For the purposes of creating an annular source, however, the solutions of greatest interest are those that do not rely on the trivial zero of the Bessel function at $r=0$. Such a solution can be obtained if one concentrates not on the area between $\mathrm{r}=0$ and the first zero of $\mathrm{J}_{1}$, but rather on the area between two zeroes at finite radii (e.g. the area between the second and third zeroes of $J_{1}$ ). Considering an annular source with boundaries at $R_{\text {inner }}$ and $R_{\text {outer }}$ then gives the condition shown in Eqn. 29. This relation is satisfied by Eqn. 30 where $\mathrm{q}$ is a positive integer. The inner and outer radii of the annulus are then related through Eqn. 31. Thus, so long as the proper relationship between $R_{\text {inner }}$ and $R_{\text {outer }}$ is maintained, it is possible to create an annular discharge while maintaining the fundamental properties of the helicon source for the $\mathrm{m}=0$ mode. For reference, the $\mathrm{J}_{0}-\mathrm{J}_{2}$ Bessel functions are plotted in Fig. 2 as a function of their argument, $\mathrm{Tr}$.

$$
\begin{gathered}
\frac{\partial J_{m}(x)}{\partial x}=\frac{m}{x} J_{m}(x)-J_{m+1}(x) \\
J_{1}\left(T R_{\text {wall }}\right)=0 \\
J_{1}\left(T R_{\text {inner }}\right)=J_{1}\left(T R_{\text {outer }}\right)=0
\end{gathered}
$$




$$
\begin{gathered}
T R_{\text {inner }, \text { outer }}=\xi_{q, q+1} \\
\frac{R_{\text {outer }}}{R_{\text {inner }}}=\frac{\xi_{q+1}}{\xi_{q}}
\end{gathered}
$$

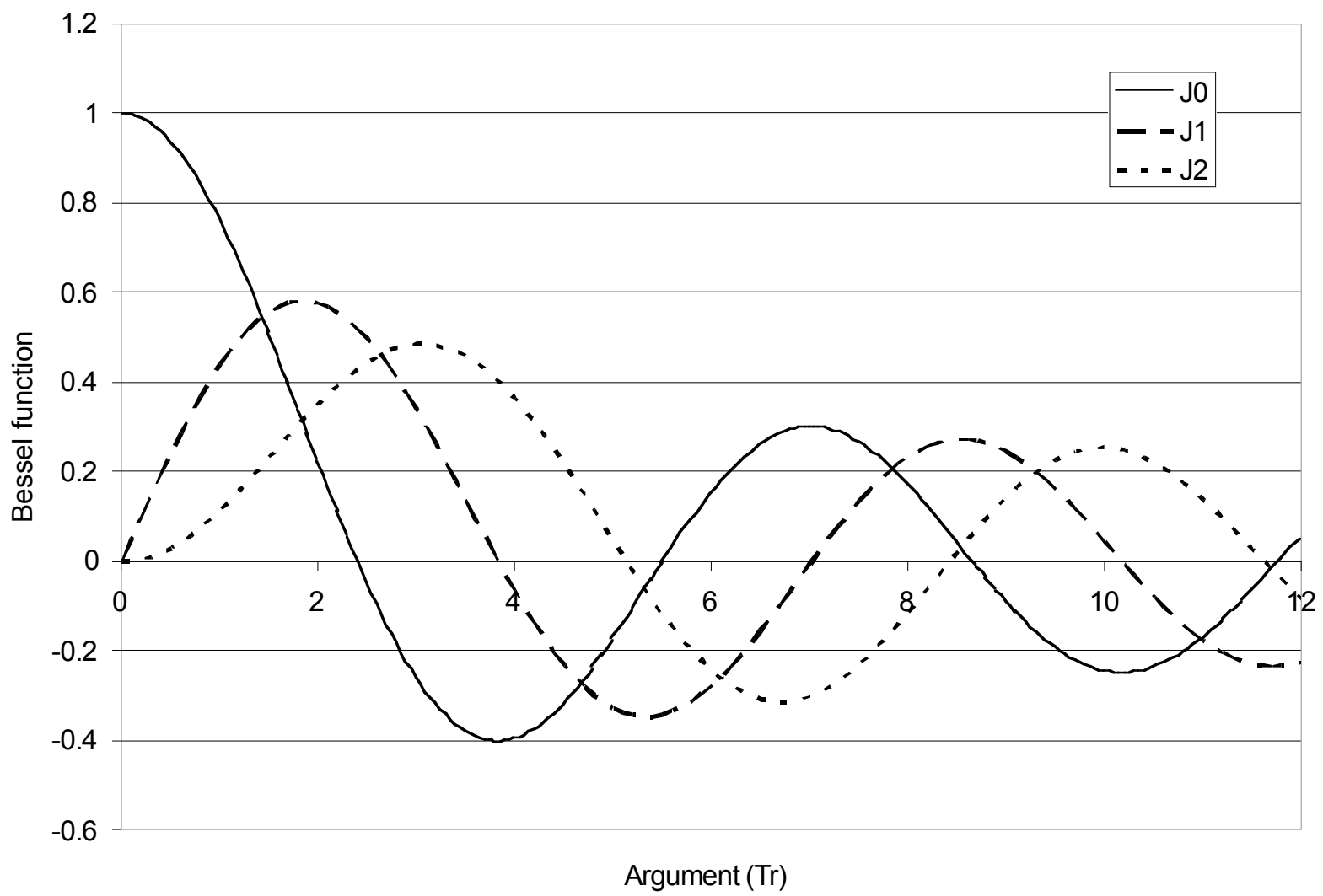

Figure 2. The Bessel functions of the first kind order 0 through 2.

We next turn our attention to the $\mathrm{m}=1$ mode. The relation shown in Eqn. 26 can be reformulated by applying the substitution $Z=\operatorname{Tr}$ and utilizing the chain rule to write the boundary condition on $\mathrm{B}_{\mathrm{r}}$ as Eqn. 32. Applying the recurrence relation of Eqn. 33 leads to Eqn. 34, where we have explicitly taken $m=1$. Finally, this equation can be solved numerically for $\mathrm{Z}=\mathrm{TR}_{\text {wall }}$ in terms of $\mathrm{k} / \alpha$. The two lowest order solutions are shown in Figure 3 and can be interpreted as giving required conditions for $\mathrm{TR}_{\text {inner }}$ and $\mathrm{TR}_{\text {outer }}$ just as Eqn. 30 did for the $\mathrm{m}=0$ mode. Taking the ratio of these curves gives the annulus ratio, $R_{\text {outer }} / R_{\text {inner }}$, needed to satisfy the boundary condition $B_{r}=0$ at the walls of an annular source for the $m=1$ wave mode. This ratio is shown in Fig. 4 and can be seen to vary with $\mathrm{k} / \alpha$. Recalling that $\alpha$ is entirely determined by $\mathrm{T}$ and $\mathrm{k}$ through Eqn. 16 reveals the fact that Fig. 4 specifies a required relationship between $R_{\text {outer }} / R_{\text {inner }}$ and $k / T$. In other words, for the $m=1$ mode, there is a unique relationship between the antenna geometry (which defines $\mathrm{k} / \mathrm{T}$ ) and the annulus geometry, and therefore the two may not be specified independently.

$$
\left.J_{m}(Z)\right|_{Z=Z_{\text {wall }}=T R_{\text {wall }}}=\left.\frac{-k Z}{m \alpha} \frac{\partial J_{m}(Z)}{\partial Z}\right|_{Z=Z_{\text {wall }}=T R_{\text {wall }}}
$$




$$
\begin{gathered}
\frac{\partial J_{m}(Z)}{\partial Z}=\frac{1}{2}\left[J_{m-1}(Z)-J_{m+1}(Z)\right] \\
J_{1}(Z)_{Z=Z_{\text {wall }}=T R_{\text {wall }}}=\frac{k Z}{2 \alpha}\left[J_{2}(Z)-J_{0}(Z)\right]_{Z=Z_{\text {wall }}=T R_{\text {wall }}}
\end{gathered}
$$

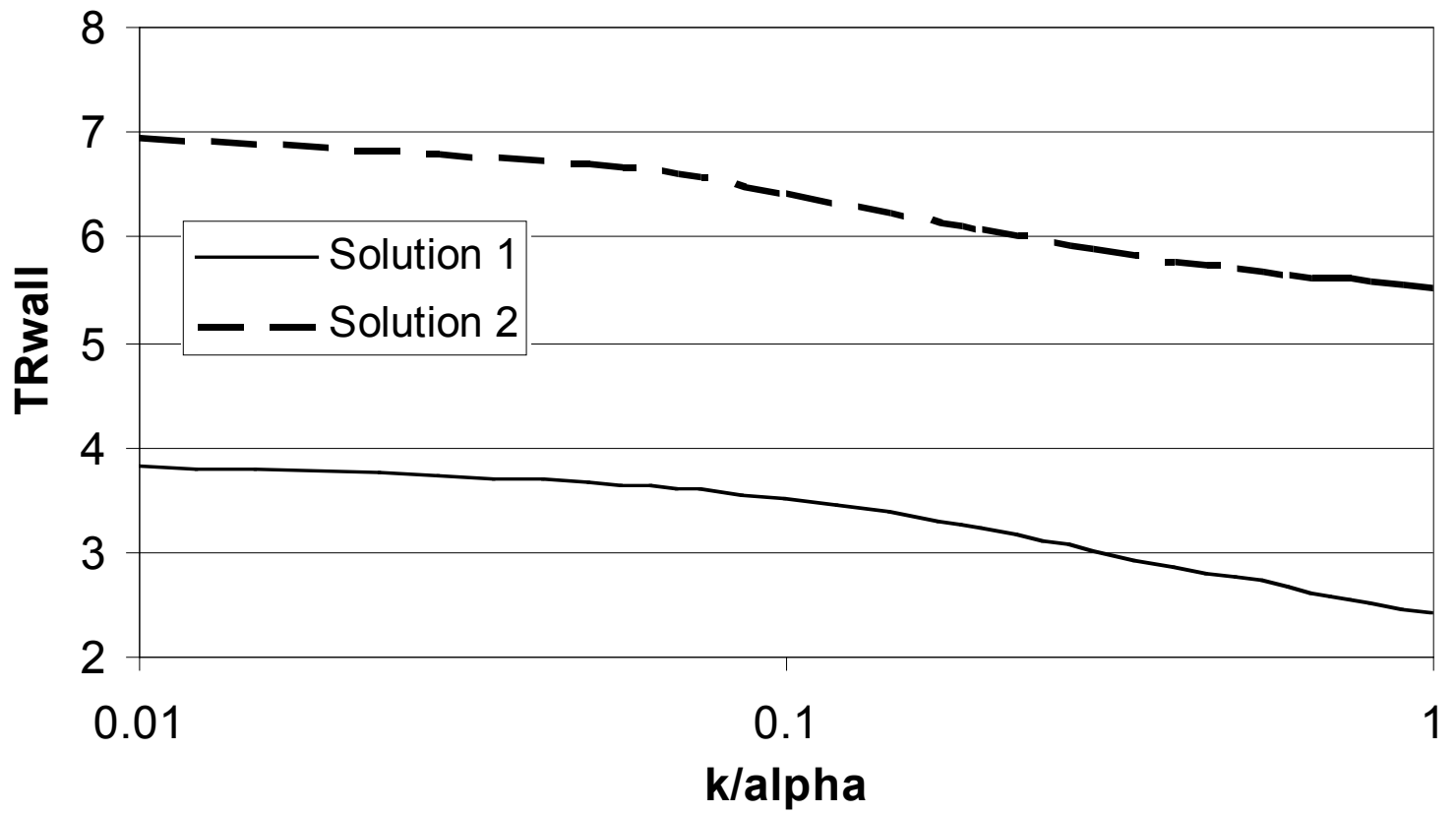

Figure 3. The two lowest order solutions to Eqn. 34 showing the relationship between $\mathbf{T R}_{\text {wall }}$ and $k / \alpha$ for the $m=1$ mode. 


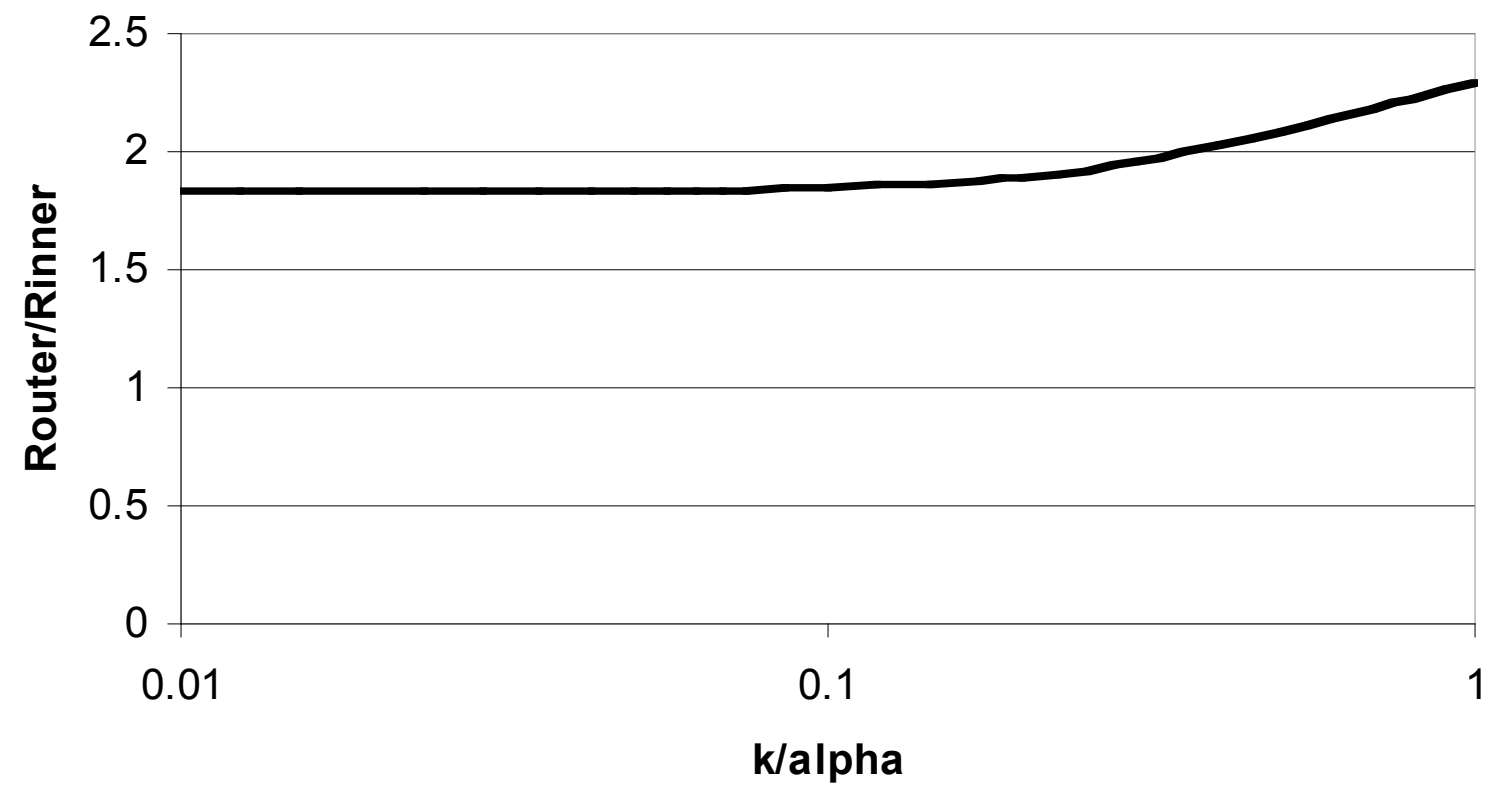

Figure 4. The relationship between annulus ratio, $\mathbf{R}_{\text {outer }} / \mathbf{R}_{\text {inner, }}$ and $k / \alpha$ for the lowest order solution of the m=1 mode.

Having derived the boundary conditions necessary to allow propagation of helicon waves in an annular channel, it is helpful to next examine the resulting wave field patterns for both the $\mathrm{m}=0$ and $\mathrm{m}=1$ modes. This is facilitated by first applying the recursion relations of Eqns. 27 and 35 to the electric field equations given by Eqns. 23 and 24 . These results are shown in Eqns. 36 and 37, which, after applying the assumed waveform $\exp [\mathrm{i}(\mathrm{m} \theta+\mathrm{kz}-\omega \mathrm{t})]$, taking the real component, and writing the result in terms of the dimensionless parameter $\mathrm{k} / \alpha$, can be expressed more conveniently as Eqns. 38 and 39. The relative magnitudes of the radial and azimuthal electric fields (i.e. the terms in square brackets in Eqns. 38 and 39) are plotted in Fig. 5, which shows the values of Tr delineating the lowest order cylindrical and annular wave modes for $\mathrm{k} / \alpha=0.2$. These same fields, including their azimuthal variation, are depicted in Figs. 6 and 7 at various values of the parameter kz- $\omega \mathrm{t}$. The existence of the solutions shown below suggests that there are no fundamental barriers to creation of annularly-bounded helicon waves so long as the boundary conditions are set appropriately.

$$
\begin{gathered}
J_{m}(x)=\frac{x}{2 m}\left(J_{m+1}(x)+J_{m-1}(x)\right) \\
E_{r}=\frac{-C_{1}}{2 T} \frac{\omega}{k}\left[(\alpha+k) J_{m-1}(T r)-(\alpha-k) J_{m+1}(T r)\right] \\
E_{\theta}=\frac{-i C_{1}}{2 T} \frac{\omega}{k}\left[(\alpha-k) J_{m+1}(T r)+(\alpha+k) J_{m-1}(T r)\right] \\
E_{r}=\frac{-C_{1}}{2 T} \frac{\omega}{k / \alpha}\left[(1+k / \alpha) J_{m-1}(T r)-(1-k / \alpha) J_{m+1}(T r)\right] \cos (m \theta+k z-\omega t)
\end{gathered}
$$




$$
E_{\theta}=\frac{C_{1}}{2 T} \frac{\omega}{k / \alpha}\left[(1-k / \alpha) J_{m+1}(\operatorname{Tr})+(1+k / \alpha) J_{m-1}(\operatorname{Tr})\right] \sin (m \theta+k z-\omega t)
$$

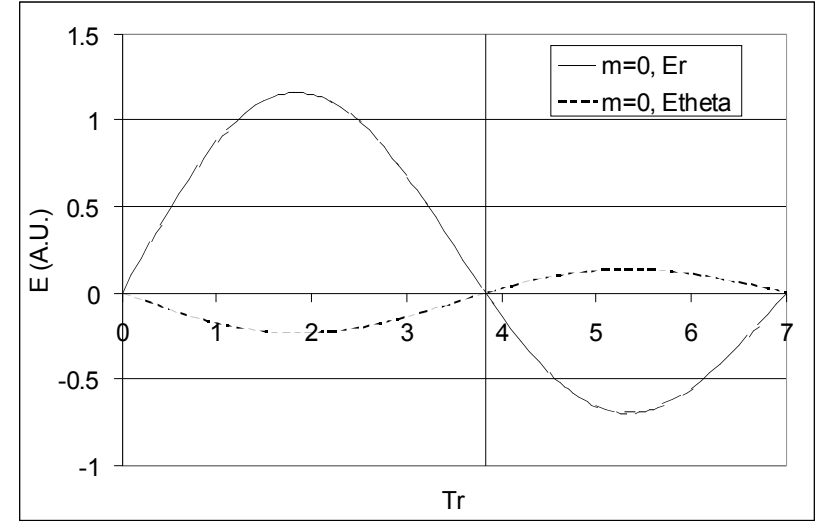

a.)

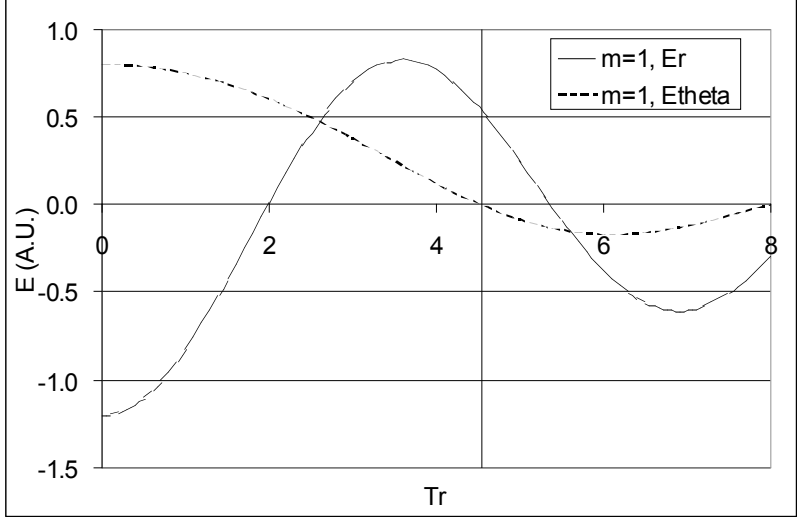

b.)

Figure 5. The relative magnitudes of the radial and azimuthal electric fields for the a.) $\mathrm{m}=0$ and $\mathrm{b}$.) $\mathrm{m}=1 \mathrm{modes}$. Values of $\mathrm{Tr}$ below the first zero of $\mathrm{E}_{\theta}\left(\right.$ or $\left.B_{\mathrm{r}}\right)$ correspond to the cylindrical solution while values above this zero represent the lowest order annular solution. 


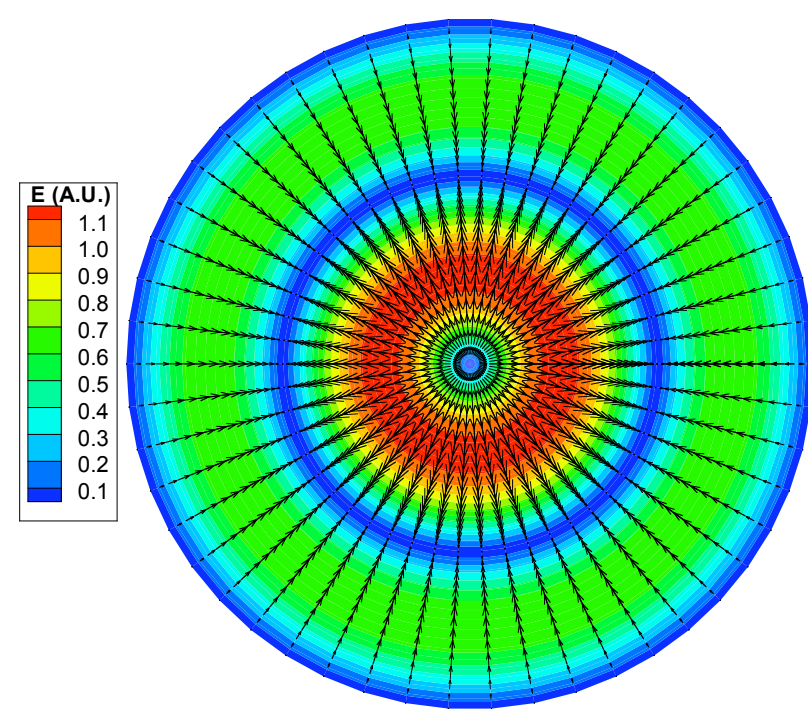

a.)

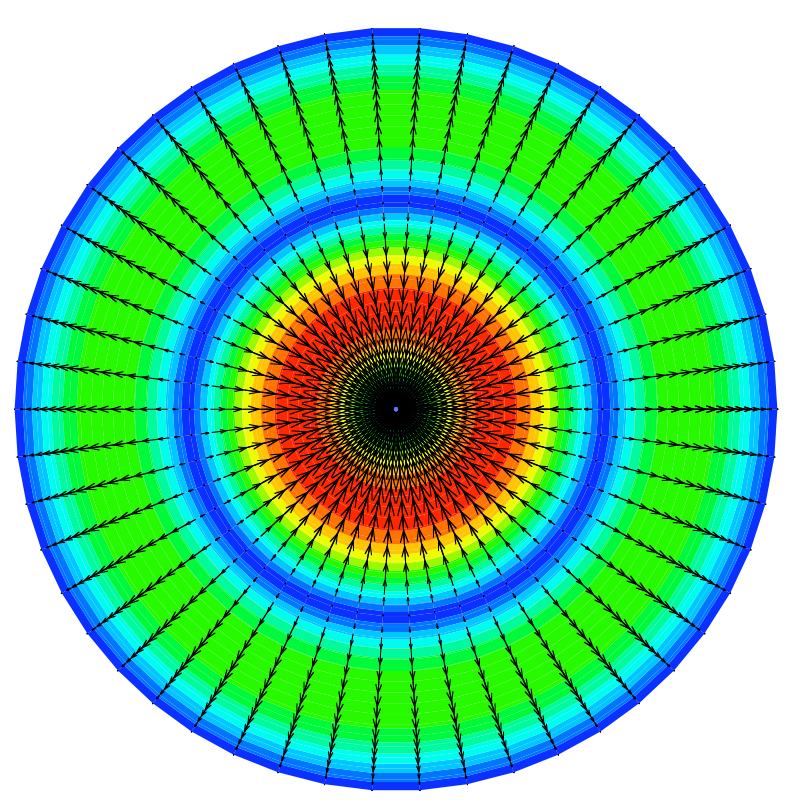

c.)

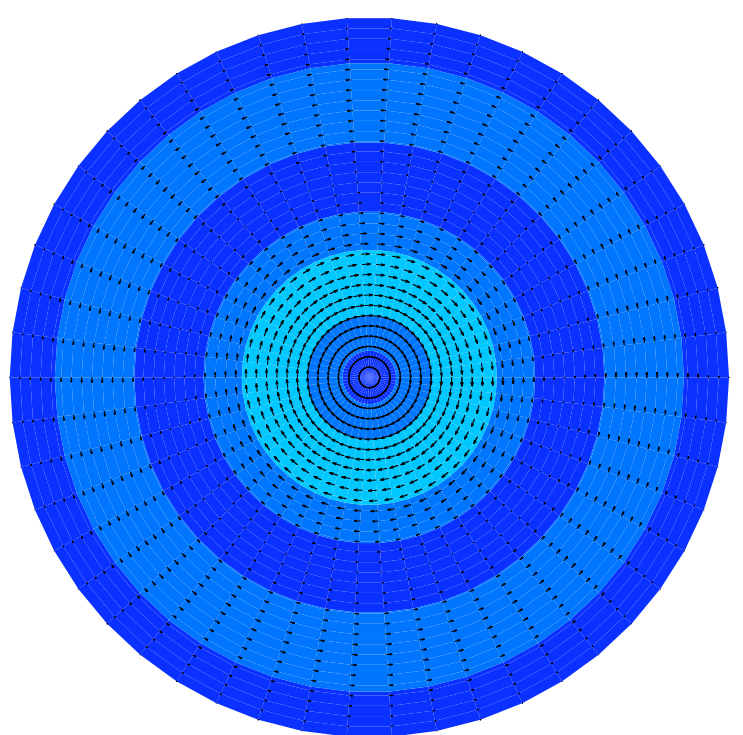

b.)

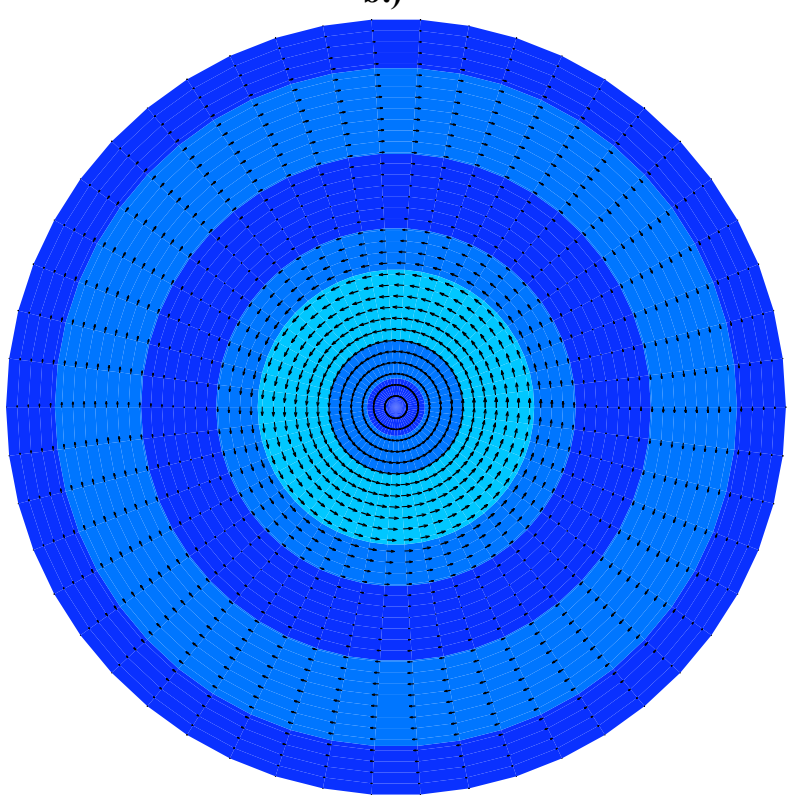

d.)

Figure 6. The electric field patterns for the $m=0$ wave mode with $k / \alpha=0.2$. Field patterns are shown at values of $\mathrm{kz}-\omega \mathrm{t}$ of a.) 0, b.) $\pi / 2$, c.) $\pi$, and d.) $3 \pi / 2$. The inner blue band in a.) depicts the boundary between the cylindrical and annular solutions. 


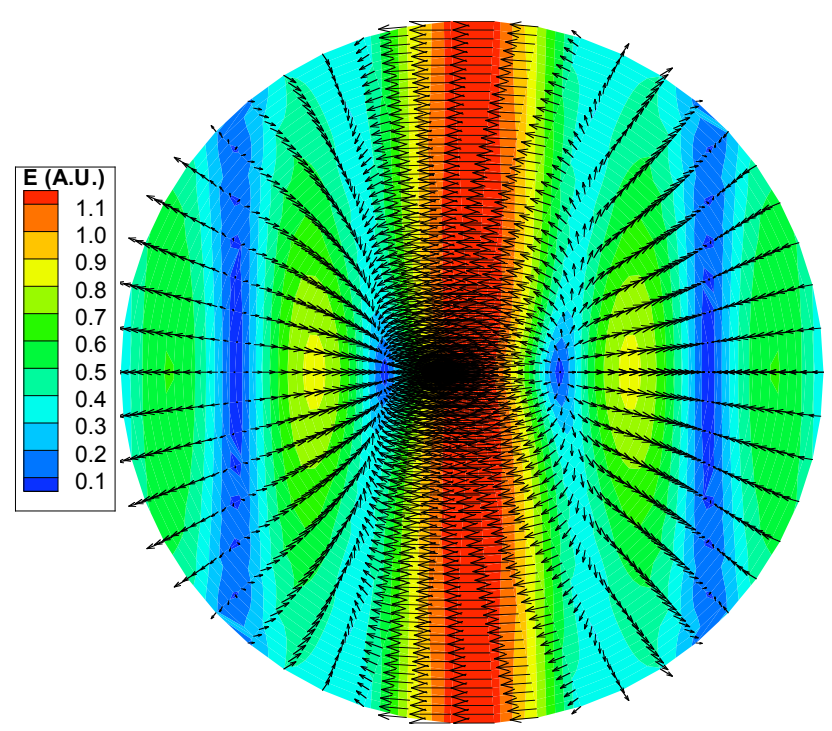

a.)

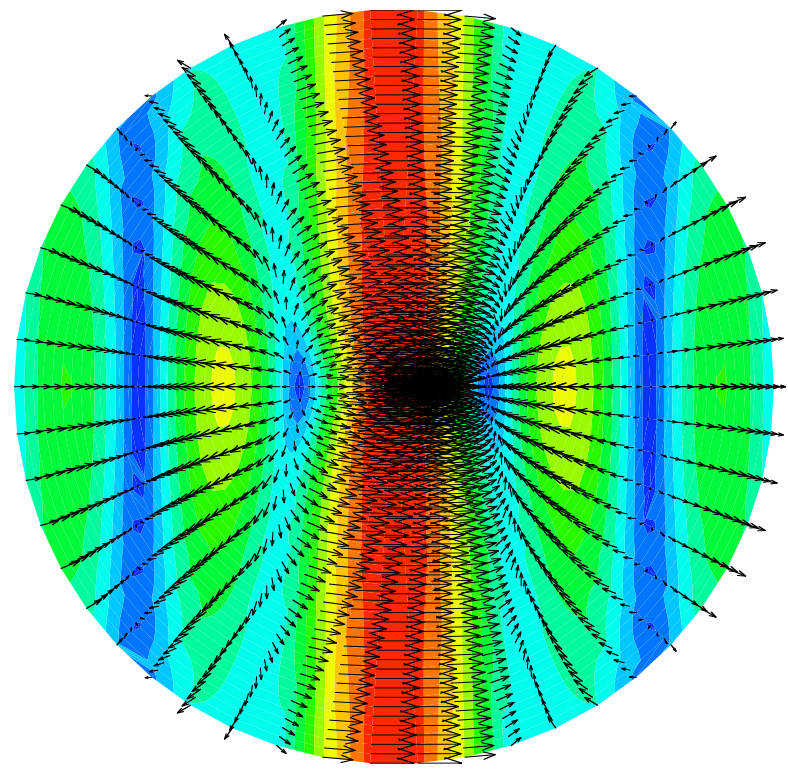

c.)

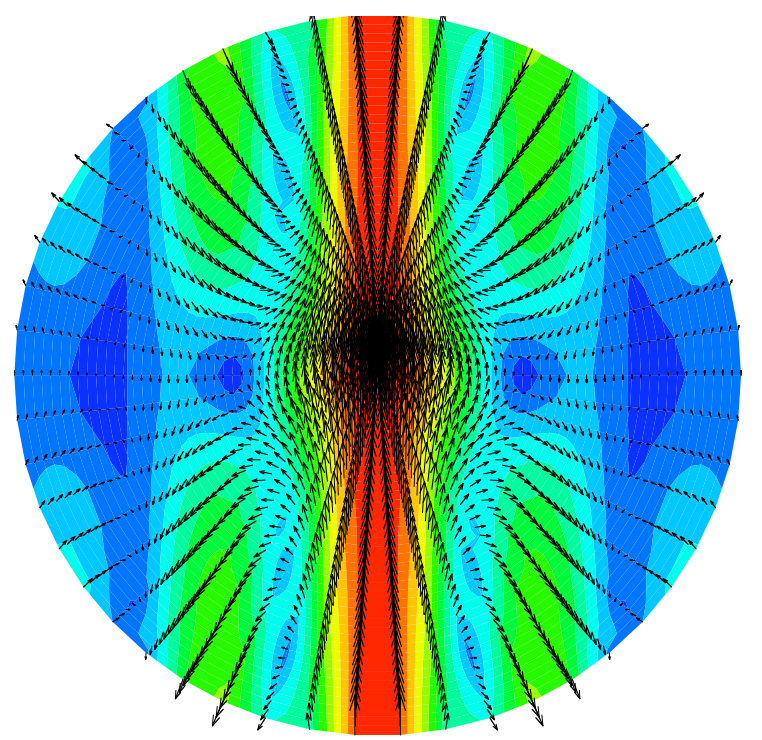

b.)

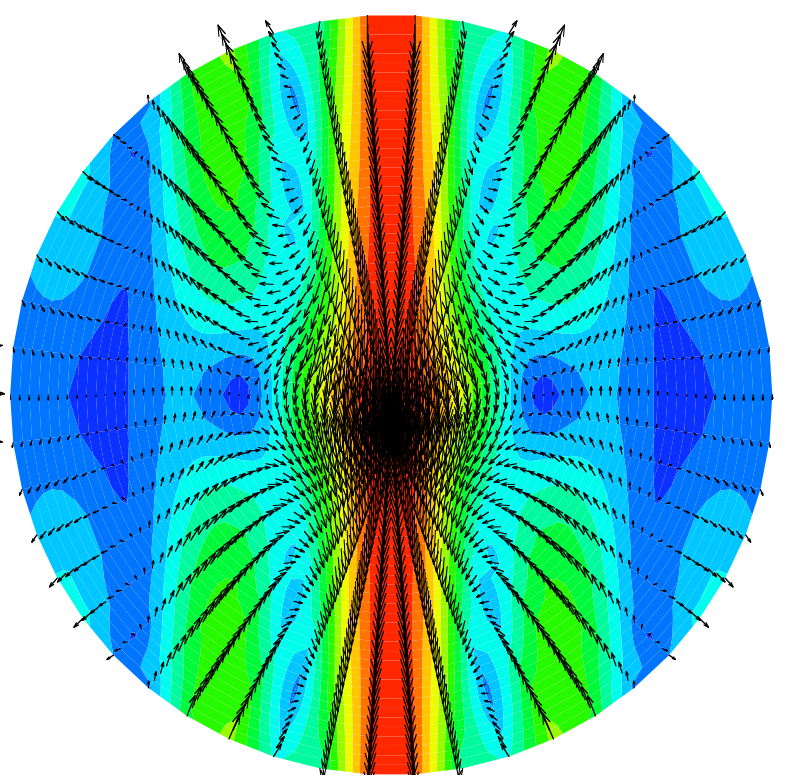

d.)

Figure 7. The electric fields patterns for the $m=1$ wave mode with $k / \alpha=0.2$. Field patterns are shown at values of

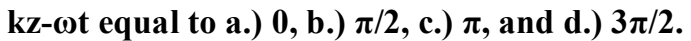

\section{Similar Work}

Near the conclusion of the work reported here, it was learned that a very similar analysis was being conducted in parallel by Yano and Walker at the Georgia Institute of Technology. The interested reader is referred to their work for an alternate approach to determining the governing relations for an annular helicon source. ${ }^{10}$ In agreement with the analysis reported in the present work, Yano and Walker concluded that it is possible to create a helicon plasma source with a coaxial configuration. ${ }^{10}$ 


\section{Conclusion}

A rigorous analysis of helicon wave physics has revealed the boundary conditions required for creation of a helicon plasma source in an annular configuration. The required boundary conditions differ between the two most common azimuthal modes, $\mathrm{m}=0$ and $\mathrm{m}=1$, showing that source geometry is fundamentally coupled to characteristics of the driving antenna. The results shown here should facilitate creation of high-density, low-temperature, coaxial helicon plasma sources that may prove beneficial as ionization stages in various EP devices, including Hall thrusters operating at high thrust-to-power ratios.

\section{Acknowledgments}

This work was performed as part of the Small Business Innovative Research (SBIR) program under contract number FA9300-05-M-3008. The authors wish to thank Dr. James Haas of the Air Force Research Laboratory, who served as the contract monitor, for support of this effort.

\section{References}

\footnotetext{
${ }^{1}$ Martinez-Sanchez, M. and Pollard, J.E., "Spacecraft Electric Propulsion - An Overview," Journal of Propulsion and Power, Vol. 14, No. 5, Sep.-Oct. 1998.

${ }^{2}$ Zhurin, V.V., Kaufman, H.R., and Robinson, R.S., "Physics of closed drift thrusters," Plasma Sources Science and Technology, Vol. 8, pp. R1-R20, 1999.

${ }^{3}$ Chen, F.F., "Experiments on helicon plasma sources," Journal of Vacuum Science and Technology A, Vol. 10, No. 4, July-Aug., 1992.

${ }^{4}$ K. de Grys, C. Rayburn, and J. Haas, "Study of Power Loss Mechanisms in the BPT-4000 Hall Thruster," AIAA2003-5277, $39^{\text {th }}$ AIAA/ASME/SAE/ASEE Joint Propulsion Conference \& Exhibit, Huntsville, AL, July, 2003.

${ }^{5}$ Hofer, R.R. and Gallimore, A.D., "The Role of Magnetic Field Topography in Improving the Performance of High-Voltage Hall Thrusters," AIAA-2002-4111, 38 ${ }^{\text {th }}$ AIAA/ASME/SAE/ASEE Joint Propulsion Conference \& Exhibit, Indianapolis, IN, July, 2002.

${ }^{6}$ Hofer, R.R., Development and Characterization of High-Efficiency, High-Specific Impulse Xenon Hall Thrusters, Ph.D Dissertation, University of Michigan, 2004.

${ }^{7}$ Chen F.F., Introduction to Plasma Physics and Controlled Fusion, Plenum Press, New York, NY, 1984, pp. 131.

${ }^{8}$ Chen, F.F., "Helicon Plasma Sources," in High Density Plasma Sources, edited by Oleg A. Popov, Noyes Publications, Park Ridge, NJ, Chap. 1, 1995.

${ }^{9}$ Chen, F.F., "Plasma Ionization by Helicon Waves," Plasma Physics and Controlled Fusion, Vol. 33, No. 4, pp. 339-364, 1991.

${ }^{10}$ Yano, M. and Walker, M.L.R., "Plasma ionization by annularly-bounded helicon waves," Physics of Plasmas, accepted for publication.
} 